\section{Character rounding}

Alpha-numeric characters produced as described in this book would look coarse. To overcome this, a technique known as character rounding is adopted. One method of achieving this is described in Appendix 3, on page 94.

\section{A complete system}

So far, in this book, a system has been described for generating text video signals to form alpha-numeric and graphic characters on a TV screen or similar video display unit.

In order to do this, the codewords stored in the RAM are 'read out' to the character generator at the correct time.

This is accomplished by three read address counters: the character counter, the row counter and the decade counter.

In order to have a complete system, in addition to the

circuitry already described, a 'source' of codewords and a write address generator are required.

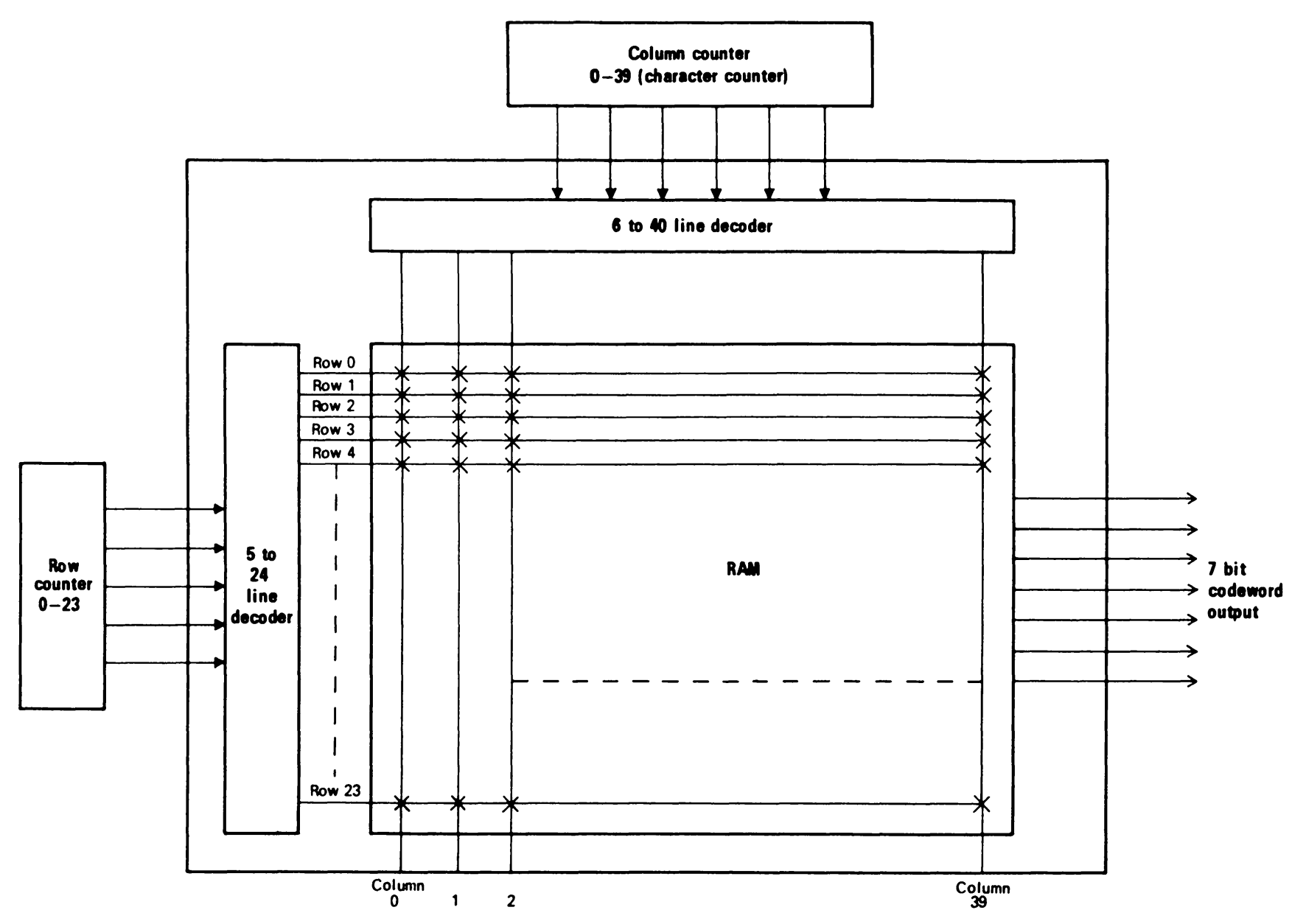

